\title{
Facial Nerve Schwannoma: The Rare/Great Mimicker of Vestibular Schwannoma/Neuroma
}

\author{
Deepthi Pathapati ${ }^{1}$ Kiran Barla ${ }^{1}$ Monal Dayal ${ }^{2}$ Rajitha Gati ${ }^{1}$ Praveen Kumar Lakota ${ }^{1}$
}

${ }^{1}$ Department of Radiodiagnosis, Omega Hospitals, Hyderabad,

Address for correspondence Deepthi Pathapati, Omega Telangana, India

2Department of Pathology, Omega Hospitals, Hyderabad, Telangana, India

\begin{abstract}
Keywords

- CP angle

$-\mathrm{CT}$

- contrastenhanced MRI

- facial nerve

- geniculate ganglion

- schwannoma

Schwannomas are benign tumors arising from Schwann cells which are a protective casing of nerves, composing myelin sheath and can develop in any nerve where Schwann cells are present. Most common are vestibulocochlear nerve schwannomas. Facial nerve schwannomas (FNSs) are uncommon tumors involving seventh nerve of which geniculate ganglion involvement is most common. Clinical presentations and the imaging appearances of FNSs are influenced by the topographical anatomy of the facial nerve and vary according to the segments involved. We report a case of 73-year-old man presenting with right side facial weakness of lower motor neuron type involvement. Computed tomography and magnetic resonance imaging are clinching the diagnosis. An early diagnosis is important in containing the disease facilitating early surgical intervention.
\end{abstract}

\section{Introduction}

Facial nerve schwannomas (FNSs) are rare slow-growing tumors, accounting for less than $1 \%$ of all temporal bone tumors. They are typically solitary, unilateral, and sporadic in nature. FNSs may be bilateral as part of neurofibromatosis-2 spectrum. Rarely, multiple schwannomas may involve peripheral branches of the facial nerve. Age of presentation varies from 5 to 84 years. No gender or side predilection is observed. ${ }^{1}$ They are rare benign tumors, which originate along the facial nerve. On imaging, they present as an enhancing cerebellopontine (CP) angle mass which is difficult to distinguish from vestibular schwannoma (also termed acoustic neuroma) and meningiomas. Facial nerve arises in the pons travel through the internal acoustic meatus and enters the facial nerve canal (or fallopian canal) which is Z-shaped structure. The bony canal has three segments: labyrinthine, tympanic, and mastoid (-Figs. 1 and $\mathbf{2}$ ).
The labyrinthine segment runs from the fundus of the internal auditory canal (IAC) to the geniculate ganglion. It is both the narrowest ( $<0.7 \mathrm{~mm}$ diameter) and the shortest (3-5 mm length) segment of the facial nerve. The labyrinthine segment travels anterolaterally from the IAC and superior to the cochlea until it reaches the geniculate ganglion. The first branch of the facial nerve, the greater superficial petrosal nerve (GSPN), exits anteriorly from the geniculate ganglion and runs along the anterior surface of the temporal bone into the pterygoid canal (vidian canal). ${ }^{2}$ Geniculate ganglion contains cell bodies associated with facial nerve-specialized taste and general somatic sensory fibers. Unless pathological, geniculate ganglion is not observed even after contrast administration. ${ }^{3}$ At the geniculate ganglion, the facial nerve makes a 75-degree turn posteriorly to become the tympanic segment (the first or anterior genu).

The tympanic segment runs from the geniculate ganglion to the second (or posterior) genu. Within the tympanic cavity, published online July 27,2021
DOI https://doi.org/

$10.1055 / \mathrm{s}-0041-1734356$ ISSN 0971-3026
(C) 2021. Indian Radiological Association.

This is an open access article published by Thieme under the terms of the Creative Commons Attribution-NonDerivative-NonCommercial-License, permitting copying and reproduction so long as the original work is given appropriate credit. Contents may not be used for commercial purposes, or adapted, remixed, transformed or built upon. (https://creativecommons.org/licenses/by-nc-nd/4.0/).

Thieme Medical and Scientific Publishers Private Ltd. A-12, Second Floor, Sector -2, NOIDA -201301, India 


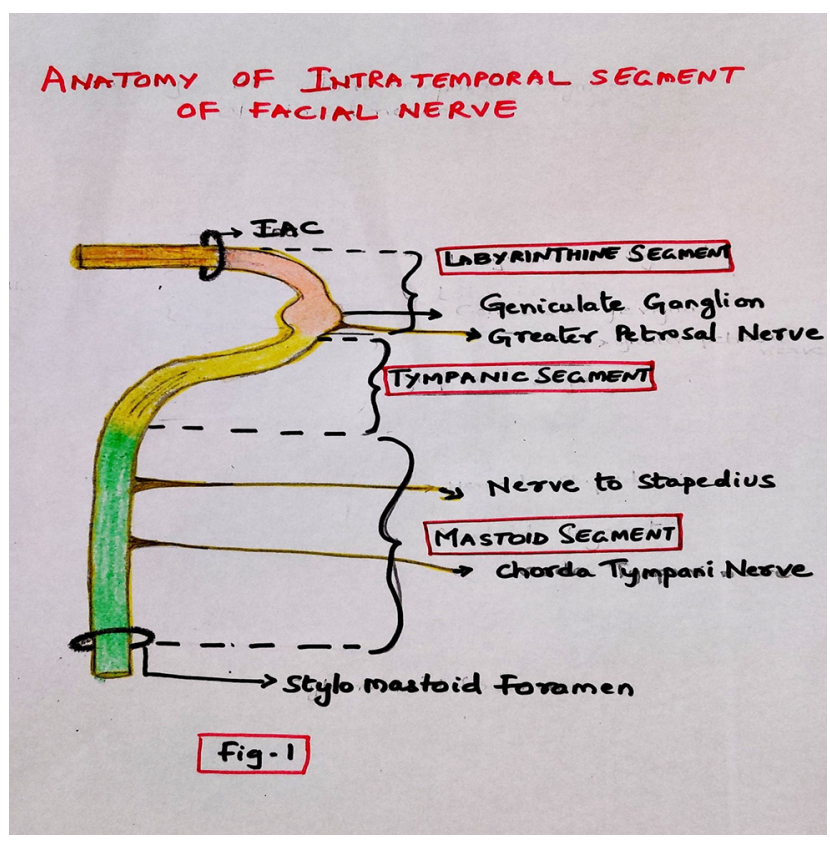

Fig. 1 Diagram of the course of the infratemporal facial nerve from the fundus of the IAC to the stylomastoid foramen. IAC, internal auditory canal.

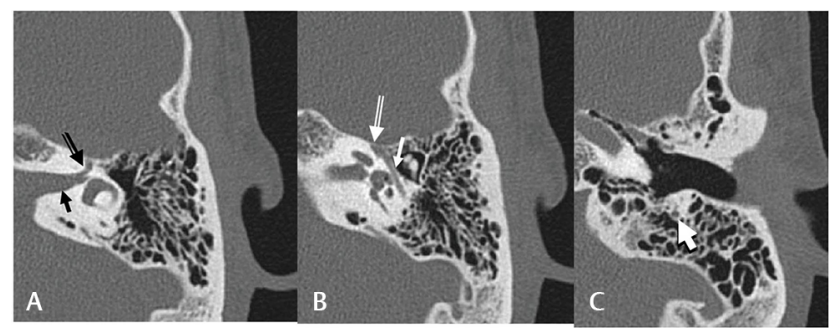

Fig. $2(\mathbf{A}-\mathbf{C})$ Axial temporal bone $C T$ images demonstrate the intracanalicular (solid arrow in [A]), labyrinthine (double-lined arrow in $[A]$ ), geniculate ganglion (double-lined arrow in $[B]$ ), tympanic (solid arrow in [B]), and mastoid (arrow in [C]) segments of the facial nerve. $\mathrm{CT}$, computed tomography.

the facial nerve passes medial to the incus. It runs posterosuperior to the cochleariform process, superior and lateral to the oval window, and then inferior to the lateral semicircular canal. At the pyramidal process, the tympanic segment turns interiorly at a 95- to 125-degree angle (at the second genu) to become the mastoid or vertical segment. The mastoid segment of the facial nerve runs posteromedially along the external auditory canal to its exit from the temporal bone at the stylomastoid foramen ( $13 \mathrm{~mm}$ in adults). The nerve exits the temporal bone at the stylomastoid foramen, entering the substance of the parotid gland. ${ }^{2}$

FNSs commonly present with peripheral facial neuropathy and/or various otologic symptoms including sensorineural and conducting hearing loss. Facial paralysis is often seen at a later stage or may be totally absent. The reason for this is attributed to the neuronal tolerance induced by the extremely slow growth of the tumor, abundant tumor vascularity, and commonly associated dehiscence of adjacent bone. Occasionally, FNSs may present as an intraparotid mass or as an intracranial lesion. ${ }^{1}$

\section{Case Report}

A 73-year-old man presented with sudden onset of right-sided facial weakness of 1-month duration. He also had a sudden onset of right-sided tinnitus without noticeable hearing impairment or dizziness. Computed tomography (CT) revealed an isodense to hyperdense lesion in the right $\mathrm{CP}$ angle with erosive extension into the petrous part of temporal bone ( - Fig. 3 ). Magnetic resonance imaging (MRI) revealed an extra-axial altered signal intensity lesion which is $\mathrm{Tl}$ hypointense and $\mathrm{T} 2$ heterogeneously hyperintense lesion in the right $\mathrm{CP}$ angle with lateral extension into IAC causing its expansion ( - Fig. 4) and another similar signal intensity lesion in the right temporal region near the petrous part of the temporal bone ( $\boldsymbol{- F i g . 5 )}$. Semicircular canals involvement was absent; however, there was a slight abutment of cochlea on the right side ( $\mathbf{- F i g . 6 A}$ ). Heterogeneous enhancement of both the lesions was noted on the contrast study ( $\mathbf{- \text { Fig. }} \mathbf{6 B}$ ).

Based on imaging findings and clinical history, diagnosis of FNS was thus conceivable. Other differential considered

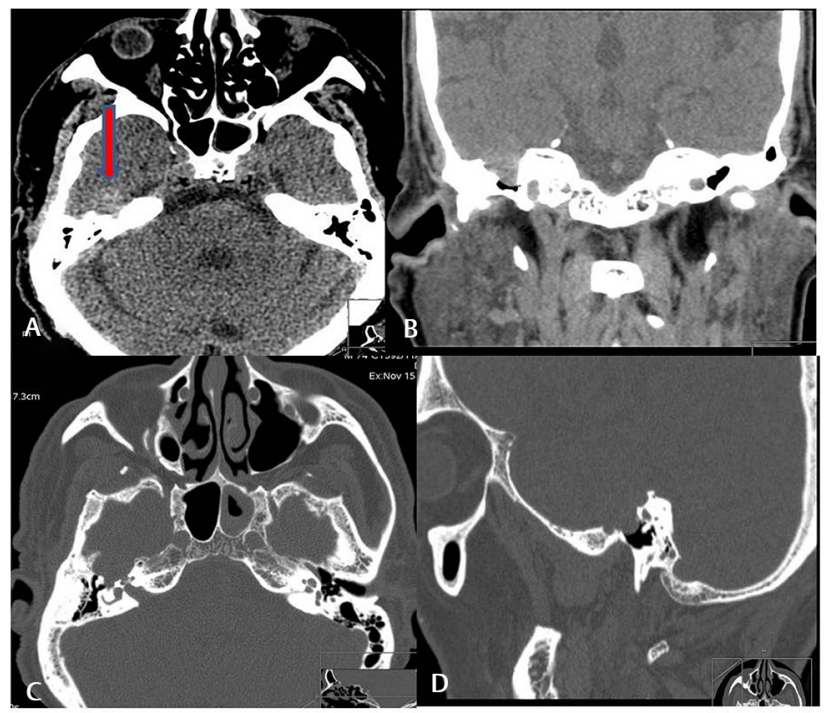

Fig. 3 (A, B) Axial and coronal reformatted images of $C T$ in soft tissue window showing hyperdense lesion in the temporal region. (C, and D) Axial and sagittal reformatted images of CT in bone window showing widening of IAC and erosion of petrous part of the temporal bone. CT, computed tomography; IAC, internal auditory canal.

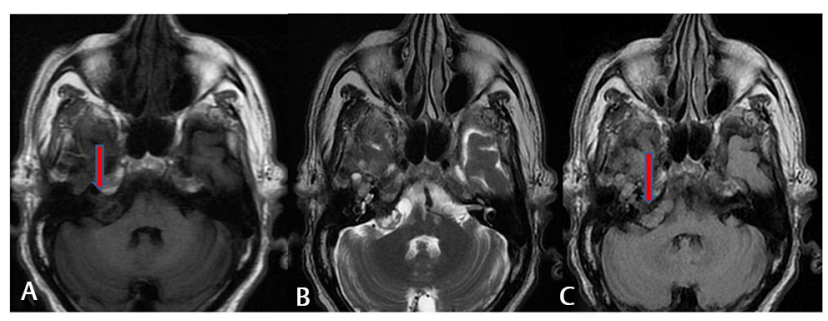

Fig. $4(\mathbf{A}-\mathbf{C})$ Axial T1, T2, and FLAIR images of MRI of the brain showing a well-defined extra-axial altered signal intensity lesion in the right $C P$ angle extending into IAC and the higher sections showing similar signal intensity lesion in the temporal region with CSF cleft sign. CP, cerebellopontine; CSF, cerebrospinal fluid; FLAIR, fluid-attenuated inversion recovery; IAC, internal auditory canal; MRI, magnetic resonance imaging. 
based on imaging was hemangioma. Cranial nerves (CNs) VII and VIII schwannomas may have identical imaging appearances if they are confined to the CP angle-IAC (CPA-IAC) complex. Hence, it is important to evaluate the findings for extended enhancement or expansion of the labyrinthine segment and geniculate ganglion of the facial nerve, as this is a subtle clue to a VII cranial than VIII nerve origin. Facial nerve decompression was done and excised tumor histopathology was reported as schwannoma (-Fig. 7).

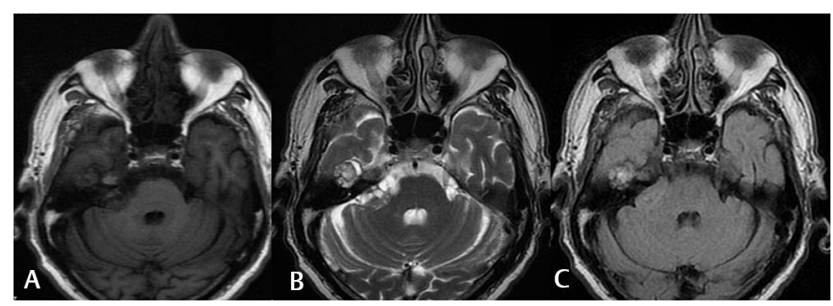

Fig. 5 (A-C) Axial T1, T2, and FLAIR images of MRI of the brain showing a well-defined extra-axial altered signal intensity lesion in temporal region with CSF cleft sign. CSF, cerebrospinal fluid; FLAIR, fluid-attenuated inversion recovery; MRI, magnetic resonance imaging.

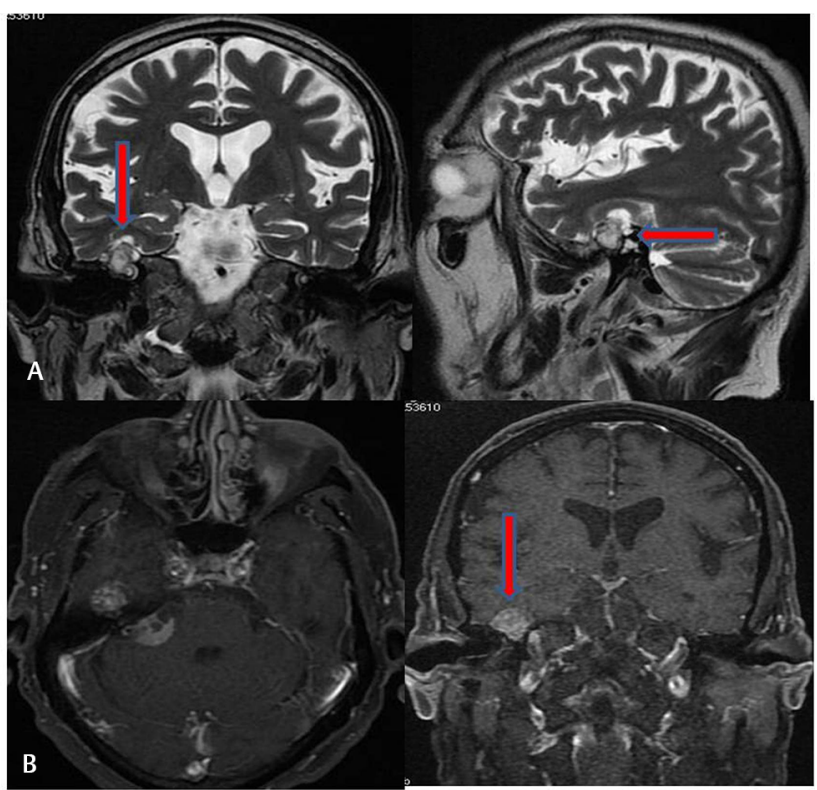

Fig. 6 (A) Sagittal and coronal reformatted MRI T2W images showing heterogeneously hyperintense lesion extending into temporal region closely abutting the cochlea on the right side. (B) Heterogeneous enhancement of both the lesions noted. MRI, magnetic resonance imaging.

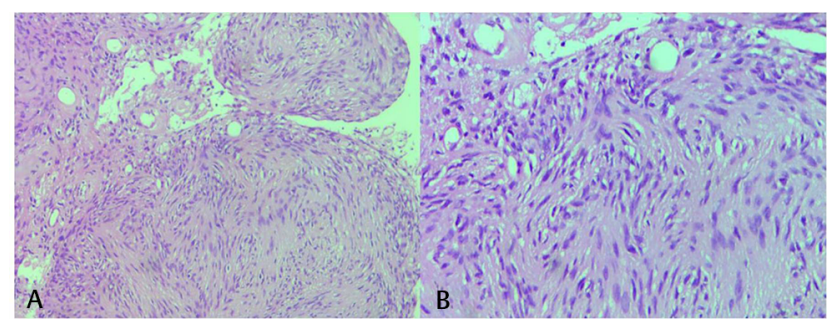

Fig. 7 (A, B) Hematoxylin and eosin-stained sections (low power field [A] and high power field [B]) showing spindle cells with ill-defined cytoplasm and wavy vesicular nucleus with focal palisading Verocay bodies.

\section{Discussion}

Schwannoma is an ectodermal benign encapsulated tumor arising from Schwann cells. ${ }^{4}$ FNSs are rare lesions that can arise anywhere along the course of the facial nerve, from its origin in the CP angle to its extracranial ramifications in the parotid space of the extracranial head and neck. Additional distinct imaging appearances were seen along the course of the facial nerve. CPA-IAC FNSs are indistinguishable from acoustic schwannomas if they did not enter the labyrinthine segment of the facial nerve canal. When extensive, a CPA-IAC FNS can present with an unusual but distinctive imaging appearance, a "dumbbell" shape due to extension from the IAC fundus through the labyrinthine segment and into the geniculate fossa. This configuration along with Tl MR enhancement and sharply scalloped, or fusiform, enlargement of the facial nerve canal is diagnostic of an FNS.

FNSs centered in the geniculate fossa most commonly conformed to the classic tubular description of the lesion or were seen as round masses enlarging only the fossa itself. When an FNS extended along the GSPN, a round middle cranial fossa extra-axial mass was seen. Lesions arising from the geniculate ganglion will have "bulbous" enlargement at the geniculate fossa itself. FNSs emanating from the GSPN scallop the anterior margin of the geniculate fossa and the adjacent bony petrous apex.

A tympanic segment FNS often lobulated into the middle ear cavity, losing its tubular configuration. The tympanic segment of the facial nerve lacks the thick surrounding bony architecture of the labyrinthine segment of the facial nerve canal. FNSs involving this segment often do not have fusiform morphology but instead are multilobular. Mastoid segment FNS could break into adjacent mastoid air cells, thereby appearing as an aggressive tumor, especially on MRI. The mastoid segment of the facial nerve is surrounded by fragile, thin-walled septations separating the mastoid air cells. When the mastoid segment of the facial nerve is involved, irregular, "invasive" tumor margins may be seen on MRI. CT explains these margins by showing the FNS breaking into surrounding mastoid air cells. ${ }^{5}$

One study of 600 temporal bone studies reported FNS incidence of $0.8 \%$. The incidence of infratemporal facial neuromas was $0.8 \%$ in a cadaveric study, although this figure is higher than the rate of clinical presentation. ${ }^{6}$ Clinical presentation not only differs on the basis of site but also by the variability of structures getting involved. Facial nerve paralysis, hearing loss, facial nerve pain, and hemifacial spasm decreased lacrimation. Slowly progressive or sudden facial weakness, often preceded by facial twitching, is a common complaint. ${ }^{7}$ In 5\% of patients with Bell's palsy, facial nerve neuroma was found to be the cause ${ }^{8}$ and normal facial nerve function was reportedly found in $27 \%$ of patients. ${ }^{9}$

Other differentials considered in the evaluation of intratemporal facial nerve lesions are cholesteatoma, facial nerve hemangioma, and perineural parotid malignancy. In these lesions, CT shows enlargement of the intratemporal facial nerve canal. Congenital or invasive acquired cholesteatoma that involves the facial nerve canal does not enhance on postcontrast-enhanced Tl MRI, which easily distinguishes 
themselves from FNS. Facial nerve hemangioma involves both the facial nerve canal and adjacent bone, with more aggressive bony changes seen as irregular margins and/or a "moth-eaten" appearance. When an internal calcified honeycomb matrix is present (50\%), ossifying hemangioma is quite distinctive. Parotid malignancy with perineural spread can be seen extending cephalad from an intraparotid invasive mass from distal to proximal along an enlarged facial nerve canal. $^{5}$

Diagnostic workup includes audiometry (audiological test), auditory brain stem evoked response audiometry, CT, and contrast-enhanced MRI.

Treatment includes surgical removal. Approach depends on the site of tumor, size of tumor, and hearing loss. Timing for surgery is controversial because facial nerve neuromas almost always grow slowly ${ }^{10}$ and waiting to pursue surgery until the facial nerve either shows progressive deterioration or becomes paralyzed in patients with normal facial nerve function has been advocated. ${ }^{11,12}$ To delay the need for complete resection further, wide tumor decompression to allow the tumor to expand outside the natural bony confines has also been advocated..$^{10}$ However, some authors believe that as the surgical approach becomes more difficult, the likelihood of postoperative complications increases, and recovery of facial function is poorer if the tumor continues to grow. The risk of surrounding structures such as the inner ear being invaded increases and eventually the brain stem may become compressed. Surgical resection of facial nerve neuromas is indicated without delay for patients with progressive facial palsy or paralysis, for large $\mathrm{CP}$ angle tumors compressing the brain stem or producing hydrocephalus, and for tumors invading the inner ear. ${ }^{10}$

\section{Conclusion}

Evaluation of the FNS requires a combined approach of correlating accurate clinical information with CT and MRI findings. Awareness of the imaging anatomy of the facial nerve and the characteristic CT and MRI appearances of FNS involving different facial nerve segments is crucial to arrive at the correct diagnosis. However, the possible imaging differentials at various locations must be borne in mind so as to avoid potential diagnostic pitfalls.

\section{Financial Support and Sponsorship}

Nil.

\section{Conflicts of Interest}

None declared.

\section{References}

1 Mundada P, Purohit BS, Kumar TS, Tan TY. Imaging of facial nerve schwannomas: diagnostic pearls and potential pitfalls. Diagn Interv Radiol 2016;22(1):40-46

2 Gupta S, Mends F, Hagiwara M, Fatterpekar G, Roehm PC. Imaging the facial nerve: a contemporary review. Radiol Res Pract 2013;2013:248039

3 Chen M-C, Tseng T-M, Hung S-H, Chen P-Y. Facial nerve schwannoma: a case report and review of the literature. Oncol Lett 2014;8(6):2787-2789

4 Symon L, Cheesman AD, Kawauchi M, Bordi L. Neuromas of the facial nerve: areport of 12 cases. BrJNeurosurg 1993;7(1):13-22

5 Wiggins RH III, Harnsberger HR, Salzman KL, Shelton C, Kertesz TR, Glastonbury CM. The many faces of facial nerve schwannoma. AJNR Am J Neuroradiol 2006;27(3):694-699

6 Saito H, Baxter A. Undiagnosed intratemporal facial nerve neurilemomas. Arch Otolaryngol 1972;95(5):415-419

7 Latack JT, Gabrielsen TO, Knake JE, et al. Facial nerve neuromas: radiologic evaluation. Radiology 1983;149(3):731-739

8 Okabe Y, Nagayama I, Takiguchi T, Furukawa M. Intratemporal facial nerve neurinoma without facial paralysis. Auris Nasus Larynx 1992;19(4):223-227

9 Sataloff RT, Frattali MA, Myers DL. Intracranial facial neuromas: total tumor removal with facial nerve preservation: a new surgical technique. Ear Nose Throat J 1995;74(4):244-246, 248-256

10 Angeli SI, Brackmann DE. Is surgical excision of facial nerve schwannomas always indicated. ? Otolaryngol Head Neck Surg 1997;117(6):S144-S147

11 King TT, Morrison AW. Primary facial nerve tumors within the skull. J Neurosurg 1990;72(1):1-8

12 Lipkin AF, Coker NJ, Jenkins HA, Alford BR. Intracranial and intratemporal facial neuroma. Otolaryngol Head Neck Surg 1987;96(1):71-79 